\title{
Demonstration of flexible thin film transistors with GaN channels
}

S. Bolat, Z. Sisman, and A. K. Okyay

Citation: Appl. Phys. Lett. 109, 233504 (2016); doi: 10.1063/1.4971837

View online: https://doi.org/10.1063/1.4971837

View Table of Contents: http://aip.scitation.org/toc/apl/109/23

Published by the American Institute of Physics

\section{Articles you may be interested in}

Low temperature thin film transistors with hollow cathode plasma-assisted atomic layer deposition based GaN channels

Applied Physics Letters 104, 243505 (2014); 10.1063/1.4884061

Improved interface properties of GaN metal-oxide-semiconductor device with non-polar plane and AIN passivation layer

Applied Physics Letters 109, 232101 (2016); 10.1063/1.4971352

III-Nitride-on-silicon microdisk lasers from the blue to the deep ultra-violet

Applied Physics Letters 109, 231101 (2016); 10.1063/1.4971357

Low p-type contact resistance by field-emission tunneling in highly Mg-doped GaN

Applied Physics Letters 109, 252101 (2016); 10.1063/1.4972408

Metal oxide semiconductor thin-film transistors for flexible electronics

Applied Physics Reviews 3, 021303 (2016); 10.1063/1.4953034

Deep ultraviolet emission from ultra-thin GaN/AIN heterostructures

Applied Physics Letters 109, 241102 (2016); 10.1063/1.4971968

\section{PHYSICS TODAY}

MANAGER'S GUIDE

WHITEPAPERS

\section{READ NOW}

PRESENTED BY

Accelerate R\&D with
Multiphysics Simulation 


\title{
Demonstration of flexible thin film transistors with GaN channels
}

\author{
S. Bolat, ${ }^{1,2, a)}$ Z. Sisman, ${ }^{1,2}$ and A. K. Okyay ${ }^{1,2,3, a)}$ \\ ${ }^{1}$ Department of Electrical and Electronics Engineering, Bilkent University, Ankara 06800, Turkey \\ ${ }^{2}$ UNAM, National Nanotechnology Research Center, Bilkent University, Ankara 06800, Turkey \\ ${ }^{3}$ Institute of Materials Science and Nanotechnology, Bilkent University, Ankara 06800, Turkey
}

(Received 25 September 2016; accepted 25 November 2016; published online 6 December 2016)

\begin{abstract}
We report on the thin film transistors (TFTs) with Gallium Nitride (GaN) channels directly fabricated on flexible substrates. GaN thin films are grown by hollow cathode plasma assisted atomic layer deposition (HCPA-ALD) at $200^{\circ} \mathrm{C}$. TFTs exhibit $10^{3}$ on-to-off current ratios and are shown to exhibit proper transistor saturation behavior in their output characteristics. Gate bias stress tests reveal that flexible GaN TFTs have extremely stable electrical characteristics. Overall fabrication thermal budget is below $200{ }^{\circ} \mathrm{C}$, the lowest reported for the $\mathrm{GaN}$ based transistors so far. Published by AIP Publishing. [http://dx.doi.org/10.1063/1.4971837]
\end{abstract}

Flexible electronic and optoelectronic devices gained a wide interest from both academia and industry due to their wide range of potential applications in areas including health monitoring, wearable electronics, and flexible displays. ${ }^{1,2}$ In conventional thin film electronics applications, amorphous Silicon (a-Si) is the most commonly utilized active material mainly because of the inexpensive manufacturing methods of the devices employing this material. ${ }^{3}$ However, the processing of the a-Si based electronics includes high temperature deposition/annealing steps, thereby hampering the possible use of the bendable substrates in this area. Therefore, there is a strong motivation in developing alternative materials which are suitable for low temperature, low cost flexible applications employing devices with high performance. In the pursuit of finding the most appropriate material for flexible electronics, several groups obtained promising results with organic semiconductors as the channel materials of the thin film transistors (TFTs). ${ }^{4,5}$ However, the long term reliability of the organic semiconductors is still under question, and this prevents them from being commercially used as the active layer material, for instance, for display applications. Alternatively, inorganic materials, mainly $\mathrm{ZnO}$ thin films and their alloyed compounds, have been widely studied and employed as the channel materials of the flexible TFTs. ${ }^{6}$ Although the reported devices have a high performance, their stability without an encapsulation layer is still an unsolved issue. ${ }^{7}$ To address this issue, alternative materials should be studied as the active layers of the TFTs on flexible substrates.

$\mathrm{GaN}$ is a widely used semiconductor, finding its commercial applications in several areas including, high power electronics, ${ }^{8}$ optoelectronics, ${ }^{9}$ and microwave electronics. ${ }^{10}$ Although having excellent electrical and optical properties, the use of this material is limited to conventional rigid substrates, mainly due to the high deposition temperature of the GaN epi-layers. ${ }^{11}$ In order to introduce this material into the low temperature applications, several methods including sputtering, ${ }^{12}$ pulsed laser deposition, ${ }^{13}$ and atomic layer deposition (ALD) $)^{14,15}$ are employed. Among the applied methods, atomic layer deposition offered the most promising

\footnotetext{
${ }^{\text {a) }}$ Author to whom correspondence should be addressed. Electronic addresses: bolat@ee.bilkent.edu.tr and aokyay@stanfordalumni.org
}

results with the demonstration of the TFTs with GaN channels fabricated with a thermal budget lower than $250^{\circ} \mathrm{C}$, the lowest processing temperature level for the nitride based electronic devices. ${ }^{16}$ Up to now, the nitride based electronic and optoelectronic devices have been realized on flexible substrates only via the transferring method, which carries the potential risk of decreasing the yield of the devices, which therefore prevents the commercialization of the proposed devices. $^{17,18}$

Here we report bottom gated TFTs with GaN channel layers directly grown on flexible polyethylene naphthalate (PEN) substrates. The overall thermal budget of the device processing steps is below $200{ }^{\circ} \mathrm{C}$, which breaks the previous self-record of lowest thermal budget GaN TFT. Devices are also fabricated on rigid substrates and their electrical properties are studied in detail.

Device fabrication steps of the TFTs on flexible substrate are summarized in Fig. 1(a) and a photograph of the flexible substrate having a series of GaN TFTs is shown in Fig. 1(b). Fabrication of the bottom gate TFT on rigid substrate starts with the RCA cleaning of the highly doped (1-5 $\mathrm{m} \Omega$-cm) p-type Si wafer. E-beam evaporation of a 200-nmthick $\mathrm{SiO}_{2}$ is performed at room temperature. The $\mathrm{SiO}_{2}$ film is patterned to define the active device areas. An HF-last clean is immediately followed by the growth of 77-nm-thick $\mathrm{Al}_{2} \mathrm{O}_{3}$ and 11-nm-thick GaN subsequently deposited at a single ALD process in a modified Fiji F200-LL ALD Reactor (Ultratech/Cambridge NanoTech Inc.), where the process temperature is kept at $200{ }^{\circ} \mathrm{C}$, and Triethylygallium (TEG) is used as the Ga precursor as suggested in Ref. 15. Active device areas are isolated by $\mathrm{BCl}_{3}$-based dry etching of the GaN layer. ${ }^{19}$ Source and drain contacts are formed by e-beam evaporation of the metal stack consisting of $\mathrm{Ti} / \mathrm{Au}$ $(30 / 150 \mathrm{~nm})$ as employed in Ref. 20. In order to keep the thermal budget of the device fabrication as low as possible, no annealing is applied after the contact metallization step. Flexible TFT fabrication starts with the solvent based cleaning of the PEN substrates. $100 \mathrm{~nm}$ thick Al thin film is deposited via thermal evaporation at room temperature, and this layer serves as the gate of the TFTs. In order to achieve the isolation between the gate and the source drain contacts, 

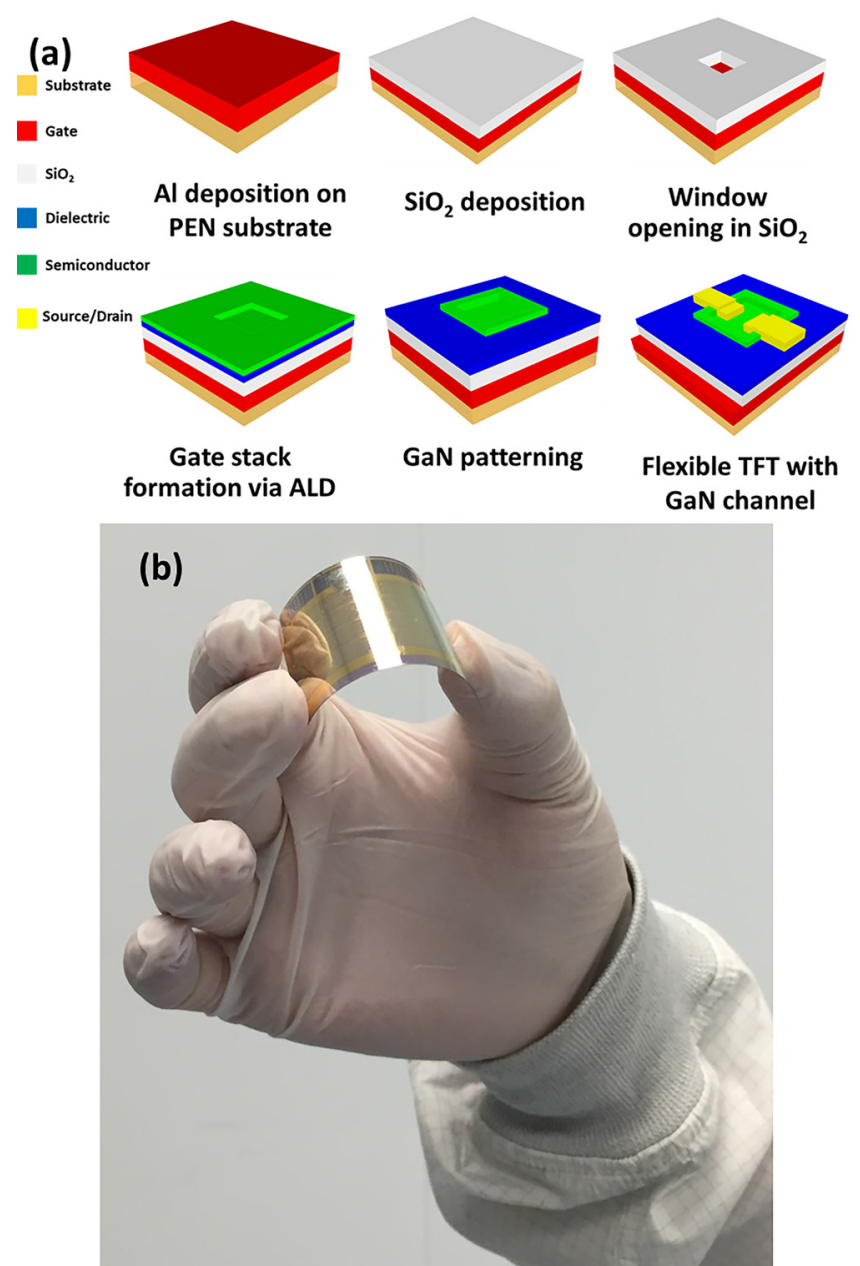

FIG. 1. (a) Schematic illustration of the device fabrication steps on flexible substrate, and (b) the photograph of the flexible substrate with a series of bottom gated TFTs with GaN channels flexed in author's hand.

$200 \mathrm{~nm} \mathrm{SiO} 2$ thin films are deposited with e-beam evaporation and patterned via lift-off technique. Afterwards, the same fabrication steps are applied with the TFTs on rigid substrates completing the production of the GaN TFTs on flexible substrates. Fabricated TFTs have $\mathrm{W} / \mathrm{L}=1$ with $\mathrm{L}=50 \mu \mathrm{m}$. Electrical measurements of the devices are performed using Keithley 4200 semiconductor parameter analyzer.

Fig. 2 shows the transfer and output characteristics of the TFTs fabricated on rigid Silicon substrates. Devices are shown to exhibit a clear saturation in their output characteristics, thereby demonstrating classical n-channel field effect transistor behavior. Several devices located at different places of the substrate are electrically characterized and on-to-off current ratios of the devices are obtained as $2 \times 10^{3}$. The threshold voltage of the device is extracted from the transfer characteristics (using $\sqrt{I_{D S}}$ ), and it is found to be $0.25 \mathrm{~V}$. Effective charge carrier mobility of the device is calculated in the linear region $\left(\mathrm{V}_{\mathrm{GS}}=10 \mathrm{~V}\right.$ and $\left.\mathrm{V}_{\mathrm{DS}}=1 \mathrm{~V}\right)$ by using equation given in (1) where the relative permittivity of the dielectric layer is obtained from a previous study ${ }^{21}$ and the mobility is found to be $0.005 \mathrm{~cm}^{2} / \mathrm{V}$-sec. This low mobility can be related to the nanocrystalline and defective nature of the ALD based GaN thin films as reported in Ref. 15

$$
\mu=\frac{I_{D S} \times L}{W \times C_{O X} \times\left(\left(V_{G S}-V_{T H}\right) \times V_{D S}-\frac{V_{D S}^{2}}{2}\right)} .
$$

Fig. 3 shows the transfer and output characteristics of the TFTs fabricated on flexible PEN substrates. Similar to the devices on rigid substrates, flexible TFTs also follow the classical n- channel field effect transistor behavior. On-to-off ratios of the devices are obtained as $7 \times 10^{2}$. The threshold voltage of the device is extracted from the transfer characteristics (using $\sqrt{I_{D S}}$ ), and it is found to be $2.5 \mathrm{~V}$. The difference in the threshold voltage can be attributed to the higher oxygen concentration in the as-deposited GaN thin films on flexible substrates (10\% Oxygen) compared to rigid substrates (2\%-3\% Oxygen), which is confirmed with the X-ray photospectroscopy measurements (not shown here). Despite the same growth conditions, the reason for higher Oxygen content in GaN films grown on flexible substrates needs a careful consideration of in-situ diffusion and thin film stress effects; however, such an analysis is beyond the scope of the current study. High oxygen concentration means higher $\mathrm{Ga}_{2} \mathrm{O}_{3}$ content in the grown $\mathrm{GaN}$ thin film; therefore the devices on the flexible substrate demonstrate a higher threshold voltage and lower on-to-off current ratio. Effective mobility of the device is calculated in the linear region $\left(\mathrm{V}_{\mathrm{GS}}=8 \mathrm{~V}, \mathrm{~V}_{\mathrm{DS}}=1 \mathrm{~V}\right)$. Calculated mobility is $0.0012 \mathrm{~cm}^{2} / \mathrm{V}$-s.

In order to realize TFTs in flexible electronics, one important step is to ensure the stable operation of the devices under bias stress. To check the stability of our devices built on flexible substrates, a gate bias stress of $10 \mathrm{~V}$ is applied while drain and source contacts are grounded. Bias duration is changed between $100 \mathrm{~s}$ and $1000 \mathrm{~s}$. This voltage stress induces an electric field of $1.3 \mathrm{MV} / \mathrm{cm}$ through the gate oxide. Fig. 4 shows the threshold voltage shift of the virgin devices after the bias stress is applied. The threshold voltage shift is an indicator of the charge trap states at the insulator
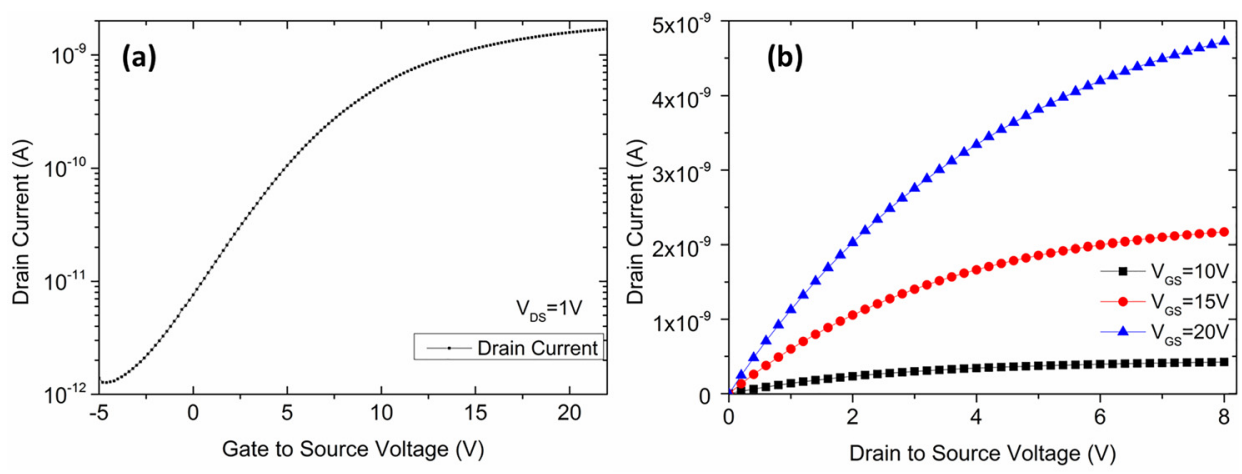

FIG. 2. (a) Transfer and (b) output characteristics of the GaN TFTs on rigid substrates. 

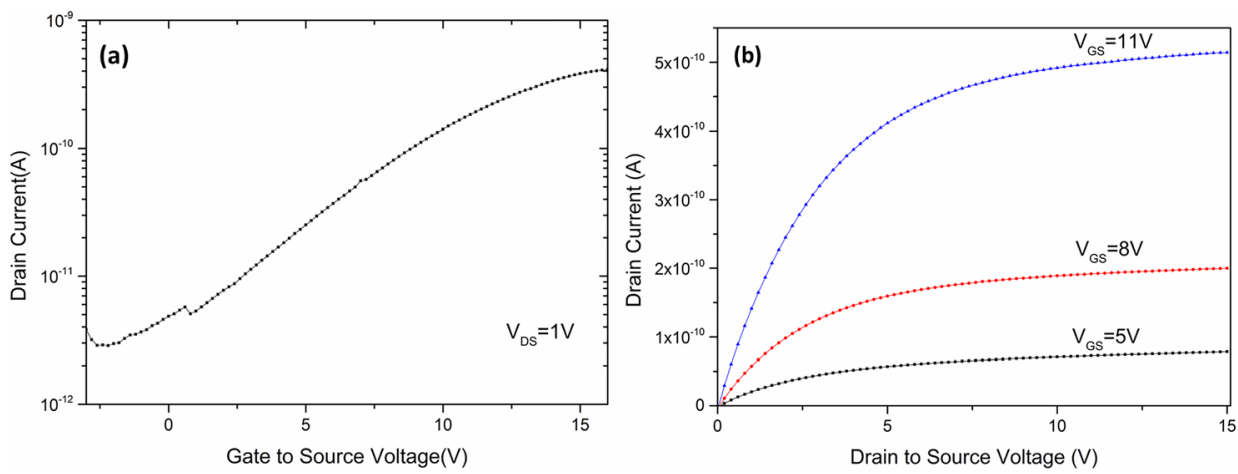

semiconductor interface and possibly those within the $\mathrm{Al}_{2} \mathrm{O}_{3}$ dielectric layer. Threshold voltage shifts to higher values with longer applied positive gate bias stress. This is mainly caused by the increased number of trapped electrons which screen the applied gate electric field resulting in an increased threshold voltage. However, the increase in the threshold voltage after $1000 \mathrm{~s}$ of bias stress is $0.14 \mathrm{~V}(\sim 5 \%)$ shift, whereas in a high performance $\mathrm{ZnO}$ based TFT, the threshold voltage shift under the same stress conditions is observed to be as high as $11 \mathrm{~V}^{7}$ which shows that the TFTs of the current work have extremely stable electrical characteristics. This result is significantly important in the sense that no encapsulation or annealing, which are commonly applied methods to electrically stabilize the TFTs with semiconducting metal oxide channels, ${ }^{22}$ are needed to stabilize the devices.

In summary, we demonstrated the fabrication of a Gallium Nitride based TFT on flexible PEN substrate. Devices on both rigid and flexible substrates are shown to exhibit a clear saturation in their output characteristics. Onto-off ratios as high as $7 \times 10^{2}$ are acquired from the characterized flexible devices. TFTs on flexible substrates are shown to have extremely stable characteristics, having threshold voltage shifts less than $0.15 \mathrm{~V}$ after the gate bias stress of $1.3 \mathrm{MV} / \mathrm{cm}$ is applied for $1000 \mathrm{~s}$. Demonstrating the operation of the GaN TFTs after direct production on flexible substrates, this study is believed to pave the way for the nitride based flexible electronics upon further materials and process optimization.

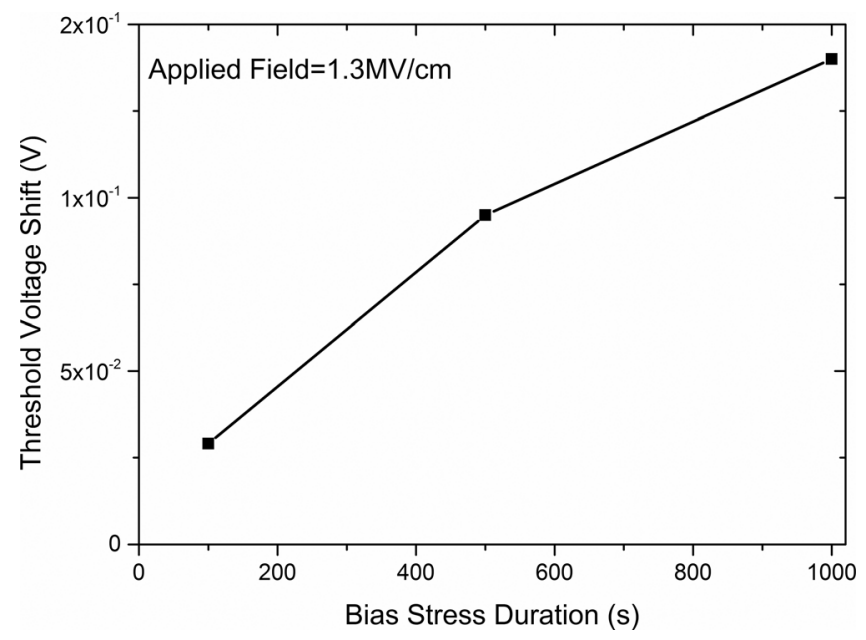

FIG. 4. Threshold voltage shift vs. positive gate bias stress of the flexible TFTs.
This work was supported in part by the Scientific and Technological Research Council of Turkey (TUBITAK), Grant Nos. 112M004, 112E052, and 113M815. A.K.O. acknowledges the support from European Union FP7 Marie Curie International Reintegration Grant (PIOS, Grant No. PIRG04-GA-2008-239444). A.K.O. acknowledges the support from the Turkish Academy of Sciences Distinguished Young Scientist Award (TUBA GEBIP), BAGEP Award, and FABED Award. S.B and Z.S. thank TUBITAK-BIDEB for Ph.D. and M.S. scholarships, respectively. Authors thank Seda Kizir for the XPS measurements.

${ }^{1}$ G. Schwartz, B. C. K. Tee, J. Mei, A. L. Appleton, D. H. Kim, H. Wang, and Z. Bao, "Flexible polymer transistors with high pressure sensitivity for application in electronic skin and health monitoring," Nat. Commun. 4, 1859 (2013).

${ }^{2}$ Y. Sun and J. A. Rogers, "Inorganic semiconductors for flexible electronics," Adv. Mater. 19(15), 1897-1916 (2007).

${ }^{3}$ R. A. Street, Technology and Applications of Amorphous Silicon (Springer, 2000).

${ }^{4}$ H. Klauk, "Organic thin-film transistors," Chem. Soc. Rev. 39(7), 2643-2666 (2010).

${ }^{5}$ C. D. Sheraw, L. Zhou, J. R. Huang, D. J. Gundlach, T. N. Jackson, M. G. Kane, I. G. Hill, M. S. Hammond, J. Campi, B. K. Greening et al., "Organic thin-film transistor-driven polymer-dispersed liquid crystal displays on flexible polymeric substrates," Appl. Phys. Lett. 80(6), 1088 (2002).

${ }^{6}$ K. Song, J. Noh, T. Jun, Y. Jung, H. Y. Kang, and J. Moon, "Fully flexible solution-deposited $\mathrm{ZnO}$ thin-film transistors," Adv. Mater. 22(38), 4308-4312 (2010).

${ }^{7}$ R. B. M. Cross and M. M. De Souza, "Investigating the stability of zinc oxide thin film transistors," Appl. Phys. Lett. 89(26), 263513 (2006).

${ }^{8}$ K. Joshin, T. Kikkawa, H. Hayashi, T. Maniwa, S. Ydkokawa, M. Yokoyama, N. Adachi, and M. Takikawa, paper presented at the Electron Devices Meeting, 2003. IEDM '03 Technical Digest (IEEE International, 2003).

${ }^{9}$ J. K. Sheu, S. J. Chang, C. H. Kuo, Y. K. Su, L. W. Wu, Y. C. Lin, W. C. Lai, J. M. Tsai, G. C. Chi, and R. K. Wu, "White-light emission from near UV InGaN-GaN LED chip precoated with blue/green/red phosphors," IEEE Photonics Technol. Lett. 15(1), 18-20 (2003).

${ }^{10}$ P. Parikh, U. K. Mishra, and Y. F. Wu, "AlGaN/GaN HEMTs-an overview of device operation and applications," Proc. IEEE 90(6), 1022-1031 (2002).

${ }^{11}$ S. Nakamura, Y. Harada, and M. Seno, "Novel metalorganic chemical vapor deposition system for GaN growth," Appl. Phys. Lett. 58(18), 2021-2023 (1991).

${ }^{12}$ R. Chen, W. Zhou, and H. S. Kwok, "Top-gate thin-film transistors based on GaN channel layer,” Appl. Phys. Lett. 100(2), 022111 (2012).

${ }^{13}$ A. Kobayashi, S. Kawano, Y. Kawaguchi, J. Ohta, and H. Fujioka, "Room temperature epitaxial growth of m-plane $\mathrm{GaN}$ on lattice-matched $\mathrm{ZnO}$ substrates," Appl. Phys. Lett. 90(4), 041908 (2007).

${ }^{14}$ C. Ozgit-Akgun, E. Goldenberg, A. K. Okyay, and N. Biyikli, "Hollow cathode plasma-assisted atomic layer deposition of crystalline AlN, GaN and $\mathrm{Al}_{\mathrm{x}} \mathrm{Ga}_{1-\mathrm{x}} \mathrm{N}$ thin films at low temperatures," J. Mater. Chem. C 2(12), 2123-2136 (2014). 
${ }^{15}$ M. Alevli, N. Gungor, A. Haider, S. Kizir, S. A. Leghari, and N. Biyikli, "Substrate temperature influence on the properties of $\mathrm{GaN}$ thin films grown by hollow-cathode plasma-assisted atomic layer deposition," J. Vac. Sci. Technol., A 34, 01A125 (2016).

${ }^{16}$ S. Bolat, C. Ozgit-Akgun, B. Tekcan, N. Biyikli, and A. K. Okyay, "Low temperature thin film transistors with hollow cathode plasma-assisted atomic layer deposition based GaN channels," Appl. Phys. Lett. 104(24), 243505 (2014).

${ }^{17}$ S. Y. Leea, K. Parka, C. Huhb, M. Kooa, H. G. Yooa, S. Kima, C. S. Ahb, G. Y. Sungb, and K. J. Leea, "Water-resistant flexible GaN LED on a liquid crystal polymer substrate for implantable biomedical applications," Nano Energy 1(1), 145-151 (2012).

${ }^{18}$ J. Chun, Y. Hwang, Y. S. Choi, T. Jeong, J. H. Baek, H. C. Ko, and S. J. Park, "Transfer of GaN LEDs from sapphire to flexible substrates by laser lift-off and contact printing," IEEE Photonics Technol. Lett. 24(23), 2115-2118 (2012).

${ }^{19} \mathrm{~S}$. Bolat, Electrical Properties and Device Applications of Atomic Layer Deposited ZnO and GaN Thin Films, Diss. Bilkent University, 2014.

${ }^{20}$ S. Bolat, B. Tekcan, C. Ozgit-Akgun, N. Biyikli, and A. K. Okyay, "Electronic and optical device applications of hollow cathode plasma assisted atomic layer deposition based GaN thin films," J. Vac. Sci. Technol., A 33(1), 01A143 (2015).

${ }^{21}$ M. D. Groner, F. H. Fabreguette, J. W. Elam, and S. M. George, "Low-temperature $\mathrm{Al}_{2} \mathrm{O}_{3}$ atomic layer deposition," Chem. Mater. 16(4), 639-645 (2004).

${ }^{22}$ Y. Y. Lin, C. C. Hsu, M. H. Tseng, J. J. Shyue, and F. Y. Tsai, "Stable and high-performance flexible $\mathrm{ZnO}$ thin-film transistors by atomic layer deposition,” ACS Appl. Mater. Interfaces 7(40), 22610-22617 (2015). 